Revue d'histoire de l'Amérique française

REVUE D.HISTOIRE DE L'AMÉRIQUE FRANÇAISE

\title{
Famili@, http://familia.inrs-culture.uquebec.ca/familia, banque de données de la recherche sur la famille au Québec
}

\section{Chantale Quesney}

Volume 54, numéro 4, printemps 2001

URI : https://id.erudit.org/iderudit/005582ar

DOI : https://doi.org/10.7202/005582ar

Aller au sommaire du numéro

Éditeur(s)

Institut d'histoire de l'Amérique française

ISSN

0035-2357 (imprimé)

1492-1383 (numérique)

Découvrir la revue

Citer ce compte rendu

Quesney, C. (2001). Compte rendu de [Famili@,

http://familia.inrs-culture.uquebec.ca/familia, banque de données de la

recherche sur la famille au Québec]. Revue d'histoire de l'Amérique française,

54(4), 608-610. https://doi.org/10.7202/005582ar d'utilisation que vous pouvez consulter en ligne. 
Famili@, http:/ / familia.inrs-culture.uquebec.ca/familia, banque de données de la recherche sur la famille au Québec.

Famili@ est un petit site de langue française qui, dans une facture tout à fait sympathique et conviviale, propose, pour l'essentiel, une banque de données bibliographiques de quelque 33500 titres relatifs à l'étude de la famille au Québec.

Le site est le résultat d'un projet entrepris à la fin des années 1990 par les professeurs Renée B.-Dandurant, de l'Institut national de la recherche scientifique (INRS), et Richard Cloutier, de l'Université Laval. Originellement, ces deux chercheurs avaient pour objectif de produire un État des lieux de la recherche sur la famille au Québec depuis 1980. Ils répondaient, en cela, à un concours lancé en 1995 par la Conférence des recteurs et des principaux des universités du Québec (CREPUQ) et du Conseil de développement de la recherche sur la famille au Québec (CDRFQ). À la suite de cette analyse, qui a nécessité la mise sur pied d'un fichier de 2000 notices, les concepteurs ont vu, dans la création d'un site Internet, la suite logique de leur projet initial. De fait, Internet a pour avantage de rendre facilement accessible au public le fruit de leur labeur. La banque de données répertorie ainsi des publications scientifiques parues entre 1980 et 1999 et produites dans le cadre des disciplines attachées aux sciences humaines, y compris celle de l'histoire. La majorité des œuvres listées sont en langue française, mais la banque indexe aussi les travaux publiés en anglais.

Le système autorise également l'interactivité et, donc, l'accroissement éventuel de la banque, puisque les utilisateurs sont invités à soumettre leurs propres références par le biais d'un formulaire envoyé électroniquement. Les données recueillies sont analysées, jugées puis, le cas échéant, intégrées dans la base de données selon les normes en vigueur dans les milieux scientifiques. La banque est mise à jour périodiquement. 
Pour connaître la prochaine date d'échéance pour la soumission de titres, il faut consulter la section "Ajouter une fiche».

Libre d'accès, cette banque s'adresse bien sûr aux milieux de la recherche, mais aussi aux intervenants des services publics et parapublics, aux responsables des associations familiales et du milieu communautaire, aux fonctionnaires, aux responsables des milieux religieux, aux avocats, aux psychothérapeutes, aux conseillers matrimoniaux, et autres. Les informations fournies par la banque comprennent la référence complète de l'ouvrage, mais également, ce qui est appréciable, des informations relatives aux objectifs poursuivis par le ou les auteurs, leur méthodologie ainsi qu'un bref résumé permettant de trouver rapidement les articles répondant aux besoins de l'utilisateur.

La banque s'appuie sur une liste de plus de 11000 mots clés et de près de 2800 noms pour effectuer la recherche. Malgré cette vaste liste, l'utilisateur appréciera la facilité avec laquelle il peut mener ses investigations. Il lui suffit, en effet, de fournir une liste d'au plus cinq mots pour obtenir le résultat de sa recherche. S'il le désire, il peut encore affiner sa demande en proposant, là encore, une liste d'au plus cinq mots à exclure. Les concepteurs de la banque ont visiblement opté pour la simplicité d'utilisation plutôt que pour la souplesse de la multiplicité des profils de recherches. Mais compte tenu du nombre relativement restreint des données, les paramètres de recherche proposés suffisent amplement pour l'obtention d'une réponse aux dimensions appropriées et ce d'autant plus qu'on offre la possibilité de choisir d'afficher ou non le sommaire des fiches.

Les concepteurs du site devraient néanmoins songer à ajouter la date de publication comme critère de sélection. Cela permettrait aux utilisateurs de mettre à jour leur propre liste sans avoir à refaire le processus depuis le début. Ainsi, un utilisateur désireux de compléter une recherche qu'il aurait effectuée trois ans auparavant, serait en mesure d'obtenir les titres parus depuis lors.

De même, la banque ne s'adressant pas spécifiquement aux chercheurs en histoire, elle n'offre pas la possibilité de faire des recherches par période. Mais comme, à l'heure actuelle, la banque compte moins de 200 entrées indexée avec le mot "histoire», les historiens auront vite fait de repérer les titres pertinents à leurs recherches. Quel intérêt, alors, peuvent trouver les chercheurs en histoire de la famille à utiliser une telle banque comparativement aux grandes banques de données traditionnelles telles que Amérique française ou America History and Life? Le principal avantage 
réside dans le fait que les chercheurs attachés à l'élaboration de la banque ne se restreignent pas à une liste de revues scientifiques déterminées pour l'enrichir. L'équipe s'intéresse activement à tout ce qui peut contribuer à l'étude de la famille au Québec. Ce faisant, la bibliographie comprend non seulement des thèses, mais également des brochures et des articles de revues qui ne sont pas inscrites auprès des grands répertoires habituels.

Petit, mais efficace, le site n'a pas, en effet, d'autre prétention que celle de fournir des titres pertinents à toutes les personnes s'intéressant aux études portant sur la famille au Québec et ce, en un clic de souris. Une courte liste de liens des institutions québécoises attachées au développement des études sur la famille vient compléter le tout. 